\title{
Braucht es die Spitalperipherie?
}

\author{
Noch gibt es in vielen Kantonen der Schweiz die kleinen Spitäler. Wie sind sie \\ heute strukturiert, welche medizinischen und paramedizinischen Leistungen \\ bieten sie an? Braucht es sie noch - oder sind sie nur noch ein lebender \\ Anachronismus?
}

Dominik Heim

Korrespondenz:

PD Dr. med. Dominik Heim

Chefarzt Chirurgie

Spital Frutigen

CH-3714 Frutigen

dominik.heim@spitalfmi.ch
Die Spitallandschaft der Schweiz verändert sich seit einigen Jahren ständig. Die Zeiten, wo jedes Tal und jede Region «sein» eigenes Spital hatte, sind vorbei. Spitäler wurden geschlossen, Akutspitäler wurden zu Pflegeheimen oder Spitälern mit Übergangspflege umfunktioniert, kleine Spitäler haben sich mit grösseren Institutionen zu Spitalgruppen zusammengeschlossen, Kooperationsmodelle mit Spitalzentren wurden entwickelt - die Spitallandschaft ist im Umbruch.

Entsprechend unserem föderalistischen System verfolgt jeder Kanton seine eigenen Ziele, hat seine eigenen Modelle und hat auch seine eigene Gangart.

Im Kanton Bern beispielsweise existieren von den einst 31 öffentlichen Betrieben noch 21, Bezirksspitäler wie Grosshöchstetten, Jegensdorf, Wattenwil, Herzogenbuchsee und Sumiswald haben ihre Türen als Akutspitäler geschlossen und wurden einem andern Zweck zugeführt. Alle peripheren Spitäler haben sich mit den umgebenden Regionalspitälern zu 6 Spitalgruppen und die Hôpital du Jura Bernois zusammengeschlossen. Zwischen gewissen Bezirksspitälern und den entsprechenden Regionalspitälern haben sich im perioperativen Umfeld enge Zusammenarbeiten ergeben [1]. Das Inselspital ist das universitäre Zentrumsspital.

Im Kanton Jura wurde aus den Spitälern Delémont, Porrentruy und Saignelegier das Hôpital du Jura, ein Spital an zwei Akutstandorten (Delémont und Porrentruy) und einem Ort für Übergangs- und chronische Pflege (Saignelegier) - das Hôpital du Jura ist ein «hôpital multisite». An den Wochenenden ist der Operationssaal in Porrentruy geschlossen, die diensthabenden Chirurgen der beiden Spitäler operieren dann in Delémont. Mit dem Universitätsspital Basel existiert für Disziplinen wie Neurochirurgie und Herzchirurgie ein Kooperationsvertrag [2].

Im Kanton Wallis hat der Walliser Staatsrat im Januar 2004 eine Aufteilung der medizinischen Tätigkeiten zwischen Standorten nach dem Kriterium der unvorgesehenen und planbaren
Tätigkeit beschlossen. An Wochenenden und in der Nacht sind nur noch die Operationssäle in Visp und Sion geöffnet, die Notfallstationen an den Standorten Martigny, Sierre und Brig sind aber ebenfalls während 24 Stunden besetzt. Das Gesundheitsnetz Wallis steht unter der strategischen Führung eines vom Staatsrat ernannten Verwaltungsrates und der operativen Leitung einer Generaldirektion. Es gibt heute drei Spitalzentren: Chablais mit den Standorten Monthey und Aigle, das «Valais central» mit den Standorten Martigny, Sion und Sierre und das Spitalzentrum Oberwallis mit den Spitälern Brig und Visp [3].

Andere Kantone haben andere Wege beschritten und eine doch ähnliche spitalpolitische Richtung eingenommen. Swiss knife, das offizielle Informationsorgan der Schweizerischen Gesellschaft für Chirurgie SGC, berichtet regelmässig in der Rubrik «Clinics» über solche Spitalmodelle und andere spital- oder gesundheitspolitischen Organisationsstrukturen.

So verfolgt jeder Kanton seine eigene Spitalpolitik, eines aber ist allen gemeinsam - kleinere Spitäler wurden/ werden geschlossen. Man sucht die Bündelung der Energien und Ressourcen, um mit zentralisierten Modellen möglichst der gesamten Bevölkerung einen möglichst optimalen Service zu guten Konditionen bieten zu können. Dies lässt sich heute bei den bestehenden Transportwegen und Transportmitteln in der Luft und zu Land unter normalen klimatischen Bedingungen häufig auch realisieren.

Und hinter all diesen Veränderungen im Gesundheitswesen steht ein Patient. Sei es der reguläre, «planbare» Patient oder der Notfallpatient, die die bestehenden Spitalstrukturen benötigen und benutzen. Sie sind es, die im kleinen, mittleren oder grossen Spital behandelt und gepflegt werden. Was bedeuten diese Entwicklungen für sie? Was bedeutet die noch existierende Spitalperipherie für den einzelnen Patienten und welche medizinischen Kapazitäten können ihm dort noch angeboten werden? An welche Bedingungen ist die medizinische Leistung gebunden, 
welche Anforderungen gelten heute für den Rettungsdienst, der die triagierten Patienten in die entsprechenden Spitäler bringt? Welche Möglichkeiten der Zusammenarbeit mit den grösseren Spitälern haben die peripheren Spitäler heute?

Und hinter all diesen Veränderungen im Gesundheitswesen steht auch der behandelnde Arzt, der niedergelassene Hausarzt, der mit und in dieser Peripherie arbeitet. Schätzt er diese Zusammenarbeit? Aber auch andere Aspekte gilt es bei der zunehmenden Globalisierung im Gesundheitswesen zu berücksichtigen. Wie sieht es aus mit der Ausbildung der zukünftigen Mediziner, wird da die Peripherie noch benötigt oder kann diese Ausbildung auch nur noch in den grösseren Zentren adäquat vermittelt werden?

Die Grundfrage zu all diesen Diskussionspunkten dürfte etwas vereinfacht wohl lauten:
«Ist die Spitalperipherie nur noch ein Anachronismus?»

Dieser Frage will das öffentliche Symposium «Der Notfallpatient - braucht er die Spitalperipherie» nachgehen, das am 1. September 2006 in Frutigen stattfinden wird. Wir laden Sie dazu herzlich ein.

\section{Literatur}

1 Heim D, Kohler A. Hat das Bezirksspital ausgedient? Die Rolle des Kleinspitals (im Spitalverband) am Beispiel des Bezirksspitals Frutigen. Swiss Knife 2005;1:12-3.

2 Peltzer J, Worreth M. Hôpital du Jura. Nouvelle structure cantonale où la difficile intégration d'un héritage. Swiss Knife 2005;4:12-3.

3 Arigoni M. Komplexes Netz. Die Reorganisation des Walliser Spitalwesens 1996-2005 aus Oberwalliser Sicht. Swiss Knife 2006;1:9-10.

\section{Der Notfallpatient - braucht er die Spitalperipherie?}

Freitag, 1. September 2006, 14.00-18.00 Uhr, Grosser Saal Hotel Simplon, 3714 Frutigen

Kontakte und Anmeldung: Ärztesekretariat, Spital Frutigen, Tel. 03367223 12, Fax 0336722185 ,

E-Mail: isabelle.baertschi@spitalfmi.ch

\section{Programm}

14.00 Begrüssung und Einleitung Hr. Ruedi Egli, Vizepräsident Verwaltungsrat Spitalgruppe fmi

14.05 Was ist ein Notfallpatient aus medizinischer Sicht? Dr. med. Rolf Ineichen, Klinikdirektor Psychiatrische Klinik, Münsingen

14.20 Was ist ein Notfallpatient aus gesundheitspolitischer Sicht? Prof. Dr. med. Hans Gerber, Kantonsarzt Kanton Bern

14.35 Was kann die Peripherie bieten beim medizinischen Notfall? Dr. med. Marco Negri, Chefarzt Innere Medizin, Spital Frutigen

14.45 Was kann die Peripherie bieten beim geburtshilflich/gynäkologischen Notfall? Dr. med. Walter Zumofen, Chefarzt Gynäkologie und Geburtshilfe, Spital Frutigen

14.55 Was kann die Peripherie bieten beim chirurgischen Notfall? Dr. med. Ruedi Minnig, Chefarzt Chirurgie, Spital Zweisimmen

15.05 Die Grenzen der Anästhesie in der Peripherie Dr. med. Christof Heim, Chefarzt Anästhesie und Intensivstation, Kantonsspital Münsterlingen

15.20 Anforderungen an den Rettungsdienst in der Peripherie Prof. Dr. med Peter Matter, ehem. Präsident des IVR, Davos-Platz

15.35 Diskussion Pause

16.10 Braucht es die Spitalperipherie für die Touristen? Dr. med. Christian Reuteler, Belegarzt Chirurgie Spitäler Saanen und Zweisimmen

16.20 Die Rega und die Peripherie Dr. med. Hans Jacomet, Leitender Arzt Helikoptereinsatz, Schweizerische Rettungsflugwacht, Zürich

16.35 Das Regionalspital und «seine» Peripherie PD Dr. med. Christoph Cottier, Chefarzt Innere Medizin, Regionalspital Emmental

16.50 Erwartungen des Zentrums an die Peripherie Prof. Dr. med. Heinz Zimmermann, Chefarzt Notfallzentrum, Inselspital Bern

17.05 Der Hausarzt und «seine» Peripherie Dr. med. Samuel Sidler, Allgemeine Medizin FMH, Praxis, Mühleturnen

17.20 Ist die medizinische Ausbildung in der Peripherie sinnvoll? Dr. med. Pierre Périat, Allgemeine Medizin FMH, Riehen

17.35 Podiumsdiskussion «Ist die Spitalperipherie nur noch ein Anachronismus?» Moderation: Hr. U. Gehrig, Direktor, PD Dr. D. Heim, Chefarzt Chirurgie, Frutigen

18.00 Ende des Symposiums 